Supporting Information

\title{
Mechanism of Ligand Recognition by Human ACE2 Receptor
}

\author{
Apurba Bhattarai ${ }^{1}$, Shristi Pawnikar ${ }^{1}$ and Yinglong Miao ${ }^{1 *}$ \\ ${ }^{1}$ Center for Computational Biology and Department of Molecular Biosciences, \\ University of Kansas, Lawrence, KS 66047, USA \\ * Corresponding email: miao@,ku.edu
}




\section{Materials and Methods}

\section{Ligand Gaussian accelerated molecular dynamics (LiGaMD)}

LiGaMD is an enhanced sampling computational technique that is developed to efficiently simulate ligand binding and unbinding based on the previous GaMD ${ }^{1}$. Details of the LiGaMD method has been described in the previous study ${ }^{2}$. A brief summary will be provided here.

We consider a system of ligand $L$ binding to a protein $P$ in a biological environment $E$. The system comprises of $N$ atoms with their coordinates $r \equiv\left\{\vec{r}_{1}, \cdots, \vec{r}_{N}\right\}$ and momenta $p \equiv$ $\left\{\vec{p}_{1}, \cdots, \vec{p}_{N}\right\}$. The system Hamiltonian can be expressed as:

$$
H(r, p)=K(p)+V(r)
$$

where $K(p)$ and $V(r)$ are the system kinetic and total potential energies, respectively. Next, we decompose the potential energy into the following terms:

$$
\begin{aligned}
& V(r)=V_{P, b}\left(r_{P}\right)+V_{L, b}\left(r_{L}\right)+V_{E, b}\left(r_{E}\right) \\
& +V_{P P, n b}\left(r_{P}\right)+V_{L L, n b}\left(r_{L}\right)+V_{E E, n b}\left(r_{E}\right) \\
& \quad+V_{P L, n b}\left(r_{P L}\right)+V_{P E, n b}\left(r_{P E}\right)+V_{L E, n b}\left(r_{L E}\right) .
\end{aligned}
$$

where $V_{P, b}, V_{L, b}$ and $V_{E, b}$ are the bonded potential energies in protein $P$, ligand $L$ and environment $E$, respectively. $V_{P P, n b}, V_{L L, n b}$ and $V_{E E, n b}$ are the self non-bonded potential energies in protein $P$, ligand $L$ and environment $E$, respectively. $V_{P L, n b}, V_{P E, n b}$ and $V_{L E, n b}$ are the non-bonded interaction energies between $P-L, P-E$ and $L-E$, respectively. According to classical molecular mechanics force fields ${ }^{3-6}$, the non-bonded potential energies are usually calculated as:

$$
V_{n b}=V_{\text {elec }}+V_{v d W}
$$


Where $V_{\text {elec }}$ and $V_{v d W}$ are the system electrostatic and van der Waals potential energies. Presumably, ligand binding mainly involves the non-bonded interaction energies of the ligand, $V_{L, n b}(r)=V_{L L, n b}\left(r_{L}\right)+V_{P L, n b}\left(r_{P L}\right)+V_{L E, n b}\left(r_{L E}\right)$. In LiGaMD, we add boost potential selectively to the essential ligand non-bonded potential energy according to the GaMD algorithm:

$$
\Delta V_{L, n b}(r)=\left\{\begin{aligned}
\frac{1}{2} k_{L, n b}\left(E_{L, n b}-V_{L, n b}(r)\right)^{2}, & V_{L, n b}(r)<E_{L, n b} \\
0, & V_{L, n b}(r) \geq E_{L, n b}
\end{aligned}\right.
$$

where $E_{L, n b}$ is the threshold energy for applying boost potential and $k_{L, n b}$ is the harmonic constant. The boost potential $\Delta V_{L, n b}(r)$ is added directly to the Hamiltonian in Eqn. (1) in LiGaMD. For simplicity, the subscript of $\Delta V_{L, n b}(r), E_{L, n b}$ and $k_{L, n b}$ is dropped in the following. When $E$ is set to the lower bound $E=V_{\max }, k_{0}$ can be calculated as:

$$
k_{0}=\min \left(1.0, k_{0}^{\prime}\right)=\min \left(1.0, \frac{\sigma_{0}}{\sigma_{V}} \frac{V_{\max }-V_{\min }}{V_{\max }-V_{\text {avg }}}\right) .
$$

Alternatively, when the threshold energy $E$ is set to its upper bound $E=V_{\min }+\frac{1}{k}, k_{0}$ is set to:

$$
k_{0}=k_{0}^{\prime \prime} \equiv\left(1-\frac{\sigma_{0}}{\sigma_{V}}\right) \frac{V_{\max }-V_{\min }}{V_{a v g}-V_{\min }},
$$

if $k_{0}^{\prime \prime}$ is found to be between 0 and 1 . Otherwise, $k_{0}$ is calculated using Eqn. (5).

Next, one can add multiple ligand molecules in the solvent to facilitate ligand binding to proteins in MD simulations ${ }^{7-8}$. In addition to selectively boosting the bound ligand, another boost potential could thus be applied on the unbound ligand molecules, protein and solvent to facilitate both ligand dissociation and rebinding. The second boost potential is calculated using the total system potential energy other than the non-bonded potential energy of the bound ligand in Eqn. (2) as: 


$$
\begin{gathered}
V_{D}(r)=V(r)-V_{L, n b}(r) \\
=V_{P, b}\left(r_{P}\right)+V_{L, b}\left(r_{L}\right)+V_{E, b}\left(r_{E}\right) \\
\quad+V_{P P, n b}\left(r_{P}\right)+V_{E E, n b}\left(r_{E}\right)+V_{P E, n b}\left(r_{P E}\right) . \\
\Delta V_{D}(r)=\left\{\begin{aligned}
\frac{1}{2} k_{D}\left(E_{D}-V_{D}(r)\right)^{2}, & V_{D}(r)<E_{D} \\
0, & V_{D}(r) \geq E_{D}
\end{aligned}\right.
\end{gathered}
$$

where $E_{D}$ and $k_{D}$ are the corresponding threshold energy for applying the second boost potential and the harmonic constant, respectively. This leads to dual-boost LiGaMD (LiGaMD_Dual) with the total boost potential $\Delta V(r)=\Delta V_{L, n b}(r)+\Delta V_{D}(r)$.

\section{Energetic Reweighting}

To calculate potential of mean force $(\mathrm{PMF})^{9}$ from LiGaMD simulations, the probability distribution along a reaction coordinate is written as $p^{*}(A)$. Given the boost potential $\Delta V(\vec{r})$ of each frame, $p^{*}(A)$ can be reweighted to recover the canonical ensemble distribution, $p(A)$, as:

$$
p\left(A_{j}\right)=p^{*}\left(A_{j}\right) \frac{\left\langle e^{\beta \Delta V(\vec{r})}\right\rangle_{j}}{\sum_{i=1}^{M}\left\langle p^{*}\left(A_{i}\right) e^{\beta \Delta V(\vec{r})}\right\rangle_{i}}, j=1, \ldots, M,
$$

where $M$ is the number of bins, $\beta=k_{B} T$ and $\left\langle e^{\beta \Delta V(\vec{r})}\right\rangle_{j}$ is the ensemble-averaged Boltzmann factor of $\Delta V(\vec{r})$ for simulation frames found in the $j^{\text {th }}$ bin. The ensemble-averaged reweighting factor can be approximated using cumulant expansion:

$$
\left\langle e^{\beta \Delta V(\vec{r})}\right\rangle=\exp \left\{\sum_{k=1}^{\infty} \frac{\beta^{k}}{k !} C_{k}\right\}
$$

where the first two cumulants are given by 


$$
\begin{gathered}
C_{1}=\langle\Delta V\rangle, \\
C_{2}=\left\langle\Delta V^{2}\right\rangle-\langle\Delta V\rangle^{2}=\sigma_{v}^{2} .
\end{gathered}
$$

The boost potential obtained from LiGaMD simulations usually follows near-Gaussian distribution. Cumulant expansion to the second order thus provides a good approximation for computing the reweighting factor ${ }^{1,}{ }^{10}$. The reweighted free energy $F(A)=-k_{B} T \ln p(A)$ is calculated as:

$$
F(A)=F^{*}(A)-\sum_{k=1}^{2} \frac{\beta^{k}}{k !} C_{k}+F_{c},
$$

where $F^{*}(A)=-k_{B} T \ln p^{*}(A)$ is the modified free energy obtained from LiGaMD simulation and $F_{c}$ is a constant.

\section{LiGaMD System setup, Simulation Protocol and Analysis}

LiGaMD simulations using the dual-boost scheme were performed on ligand binding to the ACE2 receptor. The X-ray crystal structure of the MLN-4760 inhibitor-bound ACE2 (PDB: 1R4L ${ }^{11}$ ) was used to prepare the simulation system (Figure S1). The N- and C-termini of the peptides were capped with the acetyl (ACE) and N-methyl amide (NME) neutral groups, respectively. The missing hydrogen atoms were added using the tleap module in AMBER ${ }^{12}$. The AMBER ff19SB force field ${ }^{13}$ were used for the protein. The MLN-4760 ligand was modeled with GAFF- $2^{14}$ force field. Atomic partial charges of ligand were obtained through B3LYP/6-31G* quantum calculations of the electrostatic potential, for which the charges were fitted using the antechamber program ${ }^{12,15}$. Each system was neutralized by adding counter ions and immersed in a cubic TIP3 $\mathrm{P}^{16}$ water box, which was extended $10 \AA$ from the receptor surface. The zinc and chloride atoms present near the active site of the crystal structure were also kept in the system and modeled 
in the simulations. A total of 10 ligand molecules (one in the X-ray bound conformation and another nine placed randomly in the solvent) were included in the system to facilitate ligand binding. This design was based on the fact that the ligand binding rate is inversely proportional to the ligand concentration. The higher the ligand concentration, the faster the ligand binds, provided that the ligand concentration is still within its solubility limit.

The built simulation system was energy minimized with $1 \mathrm{kcal} / \mathrm{mol} / \AA^{2}$ constraints on the heavy atoms of the protein and ligand, including the steepest descent minimization of 5,000 steps followed by a conjugate gradient minimization of 5,000 steps. The system was then heated from 0 $\mathrm{K}$ to $300 \mathrm{~K}$ for $200 \mathrm{ps}$. It was further equilibrated using the NVT ensemble at $300 \mathrm{~K}$ for $800 \mathrm{ps}$ and the NPT ensemble at $300 \mathrm{~K}$ and $1 \mathrm{bar}$ for $1 \mathrm{~ns}$ with $1 \mathrm{kcal} / \mathrm{mol} / \AA^{2}$ constraints on the heavy atoms of the protein and ligand, followed by 2 ns short cMD without any constraint. The LiGaMD simulations proceeded with $14 \mathrm{~ns}$ short cMD to collect the potential statistics, $64 \mathrm{~ns}$ GaMD equilibration after adding the boost potential and then 10 independent multiple length production runs (Table S1). Initial testing simulations showed that when the threshold energy for applying boost potential to the ligand non-bonded energy was set to the lower bound (i.e., $E=V_{\max }$ ), the bound ligand maintained the X-ray conformation and did not dissociate. In comparison, when the threshold energy was set to the upper bound (i.e., $E=V_{\min }+1 / k$ ), it enabled high enough boost potential to dissociate the ligand from the protein. In addition, boost applied not only to the ligand non-bonded energy but also to all the bonded energies can accelerate the molecular transitions for ligand dissociation and protein conformational change. Therefore, the threshold energy for applying the ligand boost potential was set to the upper bound in the LiGaMD_Dual simulations. Similarly, second boost potential was applied to the system total potential energy other than the ligand bonded/non-bonded potential energy to provide sufficient acceleration for sampling ligand 
rebinding. The threshold energy was set to the upper bound for rebinding part as well. LiGaMD_Dual production simulation frames were saved every 0.2 ps for analysis.

The $\mathrm{VMD}^{17}$ and CPPTRAJ ${ }^{18}$ tools were used for simulation analysis. The $2 \mathrm{D}$ PMF profiles were calculated through energetic reweighting of the LiGaMD simulations using PyReweighting toolkit $^{10}$, for which the Python scripts and tutorials can be found at http://miao.compbio.ku.edu/PyReweighting/. 2D PMF profiles of the interdomain Glu56:CA Ser128:CA atom distance and root-mean-square deviation (RMSD) of the MLN-4760 inhibitor relative to X-ray conformation were calculated to analyze conformational changes of the protein upon ligand binding. Residues Glu56 and Ser128 lie on the tip of $\alpha 4$ (subdomain I) and $\alpha 6$ helices (subdomain II), respectively. The bin size was set to 2.0 - $2.5 \AA$ for the atom distances. Similarly, 2D PMF profiles of the Glu56:CA - Ser128:CA atom distance and RMSD of subdomain I relative to X-ray conformation were calculated to characterize domain flexibility in the ACE2 receptor. The cutoff of simulation frames in one bin for 2D PMF reweighting was set to 500. Structural clustering was performed on the LiGaMD simulations based on the RMSD of the receptor as well as the ligand snapshots using hierarchical agglomerative algorithm in CPPTRAJ ${ }^{18}$. The RMSD cutoff was set to 2.0 A. The top representative snapshots were chosen and carefully matched with the low-energy wells of the PMF profiles regarding the interdomain distance and ligand RMSD reaction coordinates. These snapshots represent energy minima with ligand making stable polar interactions with the polar residues of the receptor. 


\section{LiGaMD simulation input parameters}

Example input parameters used in dual-boost LiGaMD simulations include the following in addition to those used in conventional MD:

igamd $=7, i E P=2, i E D=2$, irest_gamd $=0$,

ntcmd $=2000000$, nteb $=30000000$, ntave $=400000$,

ntcmdprep $=800000$, ntebprep $=800000$,

sigma0P = 9.0, $\operatorname{sigma0D}=9.0$,

icfe $=1$, ifsc $=1$, gti_cpu_output $=0$, gti_add_sc $=1$,

timask1 = ':600', scmask $\overline{1}=$ ':600',

timask2 = ", scmask2 =",

$i b b l i g=1, n l i g=10$, atom $\_p=7815$, atom_l $l=3$, dblig $=2.5$, 
Table S1: Summary of LiGaMD simulations performed on the ACE2 receptor in the presence of the MLN-4760 inhibitor.

\begin{tabular}{|c|c|c|c|c|c|}
\hline Simulation & ${ }^{\text {a }} \mathbf{N}_{\text {atoms }}$ & Dimension $\left(\AA^{3}\right)$ & $\begin{array}{c}\text { Simulation } \\
\text { (ns) }\end{array}$ & $\begin{array}{c}{ }^{\mathrm{b}} \Delta \mathrm{V}_{\text {avg }} \\
\text { (kcal/mol) }\end{array}$ & $\begin{array}{c}{ }^{\mathrm{c}} \sigma_{\Delta v} \\
(\mathrm{kcal} / \mathrm{mol})\end{array}$ \\
\hline Sim1 & 100,449 & $129 \times 93 \times 93$ & 2000 & 126.8 & 9.76 \\
\hline Sim2 & 100,449 & $129 \times 93 \times 93$ & 2000 & 127.6 & 9.62 \\
\hline Sim3 & 100,449 & $129 \times 93 \times 93$ & 2000 & 127.2 & 9.14 \\
\hline Sim4 & 100,449 & $129 \times 93 \times 93$ & 1600 & 129.2 & 10.3 \\
\hline Sim5 & 100,449 & $129 \times 93 \times 93$ & 1600 & 129.5 & 10.5 \\
\hline Sim6 & 100,449 & $129 \times 93 \times 93$ & 1000 & 129.3 & 10.4 \\
\hline Sim7 & 100,449 & $129 \times 93 \times 93$ & 1000 & 129.5 & 10.5 \\
\hline Sim8 & 100,449 & $129 \times 93 \times 93$ & 700 & 129.4 & 10.4 \\
\hline Sim9 & 100,449 & $129 \times 93 \times 93$ & 700 & 129.4 & 10.4 \\
\hline Sim10 & 100,449 & $129 \times 93 \times 93$ & 700 & 129.4 & 10.4 \\
\hline
\end{tabular}

${ }^{\mathrm{a}} N_{\text {atoms }}$ is the number of atoms in the simulation system.

${ }^{\mathrm{b}} \Delta \mathrm{V}_{\mathrm{avg}}$ and ${ }^{\mathrm{c}} \sigma_{\Delta \mathrm{V}}$ are the average and standard deviation of the LiGaMD boost potential, respectively. 


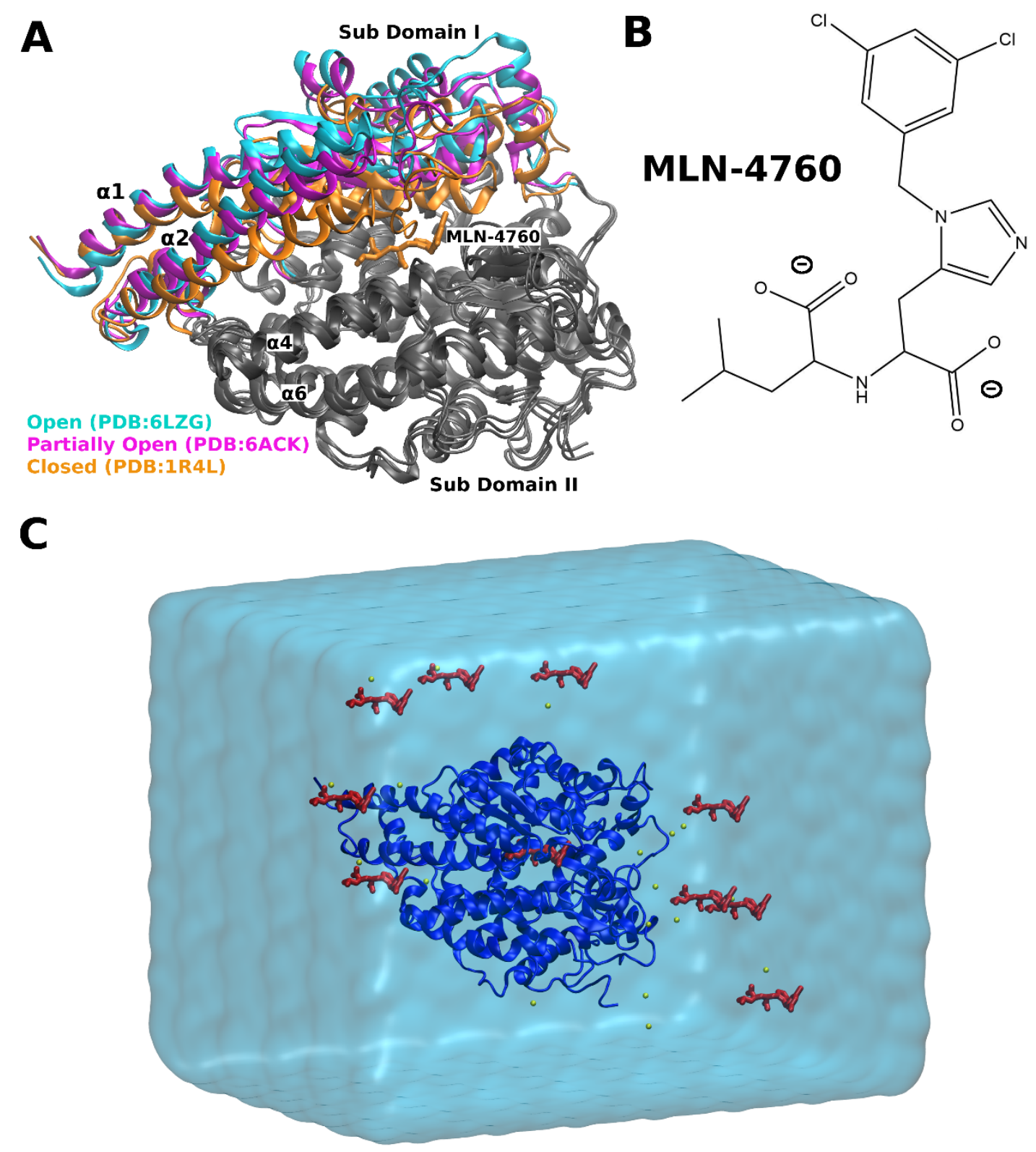

Figure S1: (A) X-ray and cryo-EM strucutres of the ACE2 receptor with subdomain $I$ in the "Open" (cyan, PDB: 6LZG), "Partially Open" (magenta, PDB: 6ACK) and "Closed" (orange, PDB: 1R4L) conformations. Subdomain II is stable and colored in gray. In the "Closed" conformation, the receptor is bound by the MLN-4760 inhibitor. The protein is shown as ribbons and the ligand as sticks (orange). (B) Structure of the MLN-4760 inhibitor molecule. (C) Computational model of the ACE2 receptor (blue ribbons) with 10 MLN-4760 ligand molecules (red sticks) (one in the X-ray bound conformation and another nine placed randomly in the solvent) used in LiGaMD simulations. The system was neutralized by adding counter ions and immersed in a cubic TIP3P water box, which was extended $10 \AA$ from the receptor surface. 


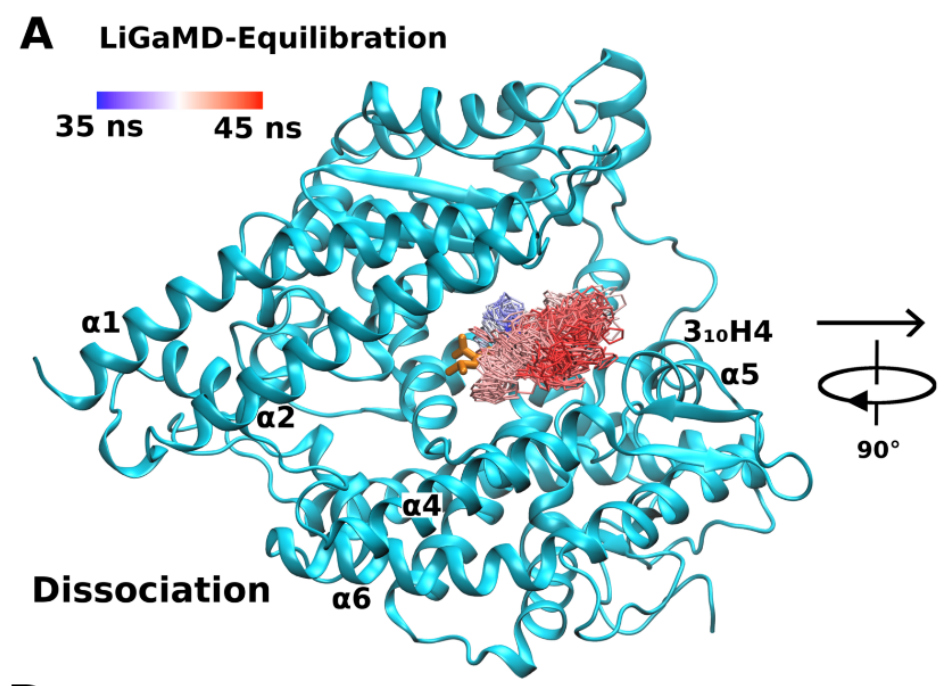

B
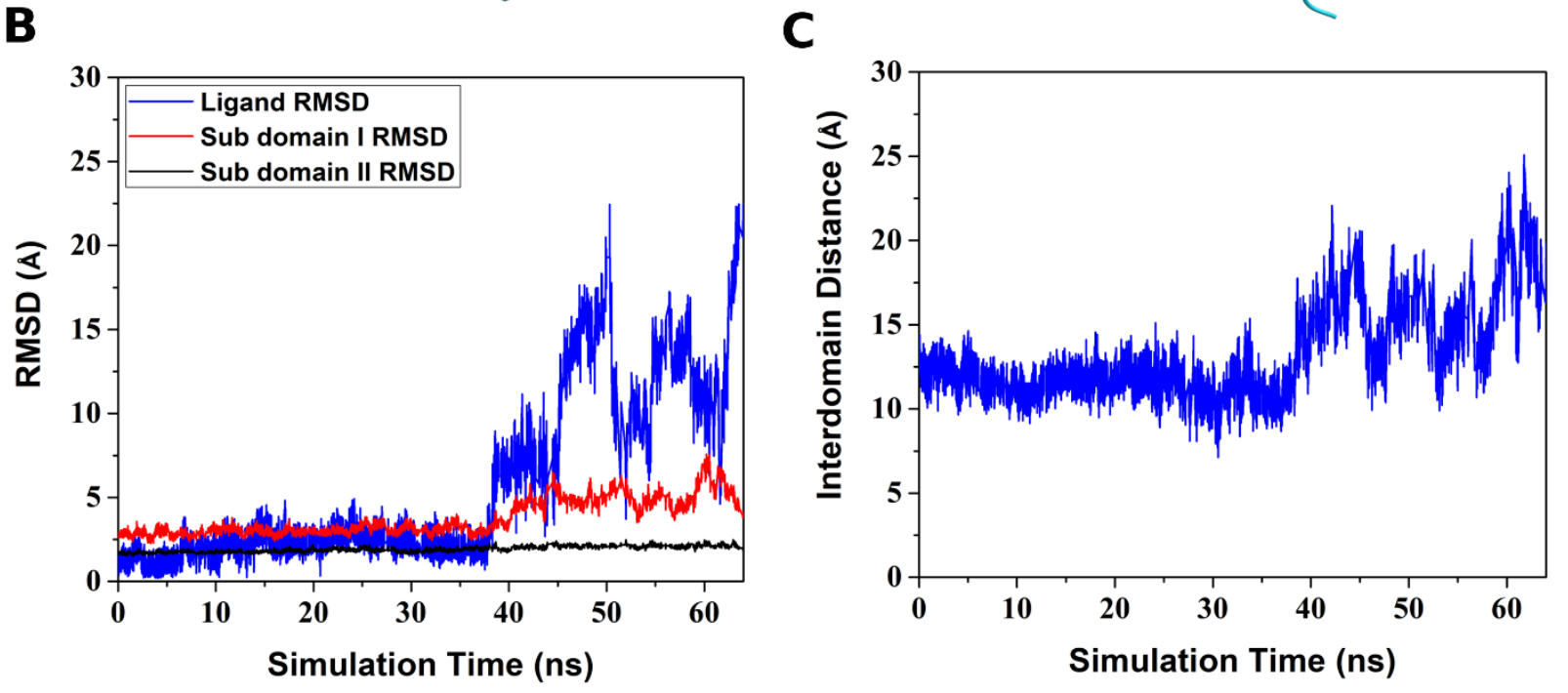

Figure S2: (A) Two views of the ligand dissociation pathway observed in LiGaMD equilibration, for which the center ring of MLN-4760 is represented by lines and colored by simulation time in a blue-white-red (BWR) color scale. (B) Time course of the MLN-4760 ligand RMSD (blue), sub domain I RMSD (red) and sub domain II RMSD (black) calculated from LiGaMD equilibration trajectory, in which the MLN-4760 ligand dissociated from the active site of the receptor. (C) Time course of the interdomain distance (Glu56:CA - Ser128:CA) calculated from LiGaMD equilibration trajectory. 

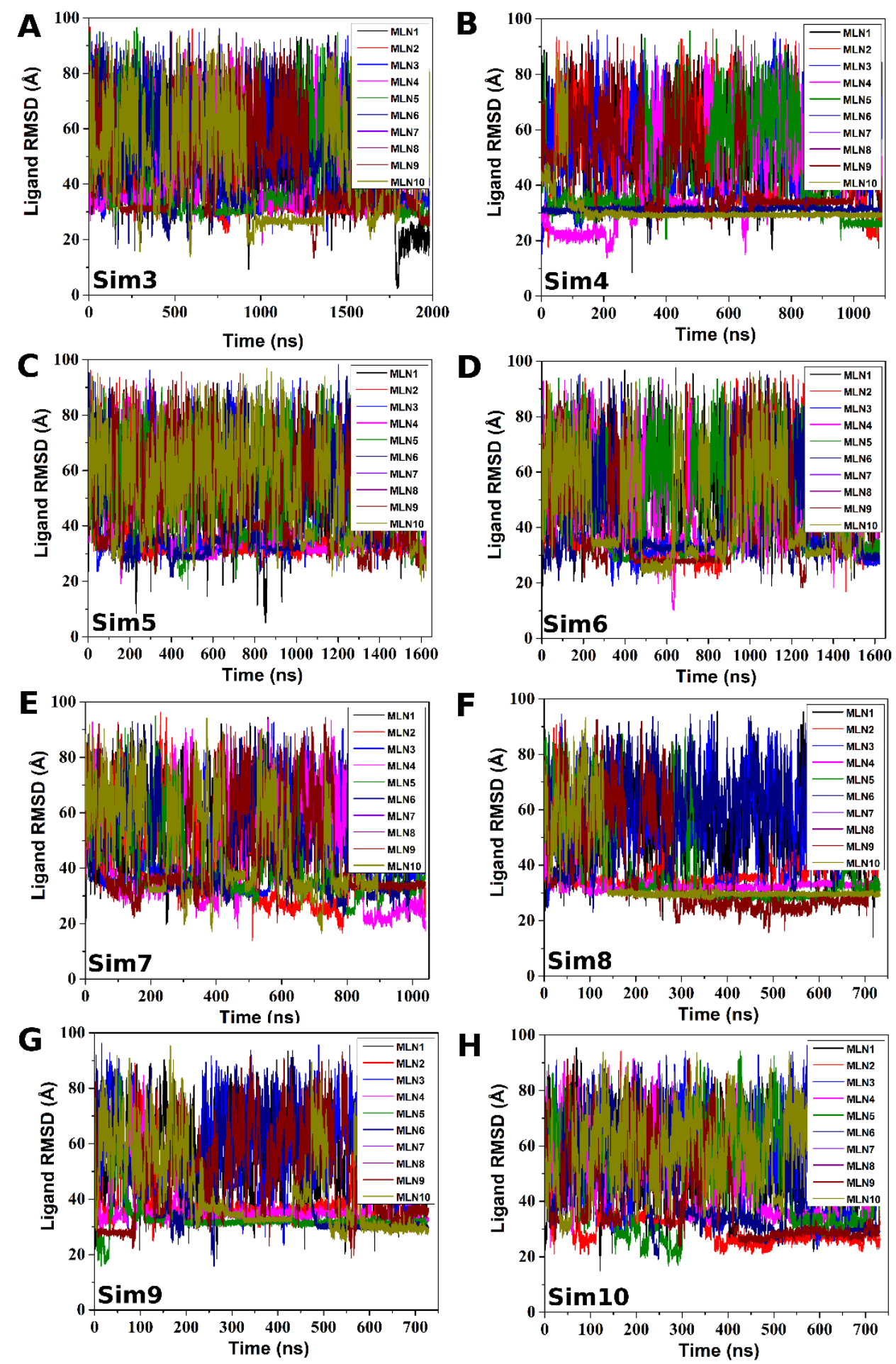

Figure S3: Time courses of the MLN-4760 ligand RMSD relative to the bound X-ray conformation (PDB: 1R4L) calculated from (A) "Sim3", (B) "Sim4", (C) "Sim5", (D) "Sim6", (E) "Sim7", (F) "Sim8", (G) "Sim9" and (H) "Sim10" LiGaMD trajectories of varying lengths ranging 700-1600 ns, in which the MLN-4760 ligand did not bind to the active site of the receptor. 


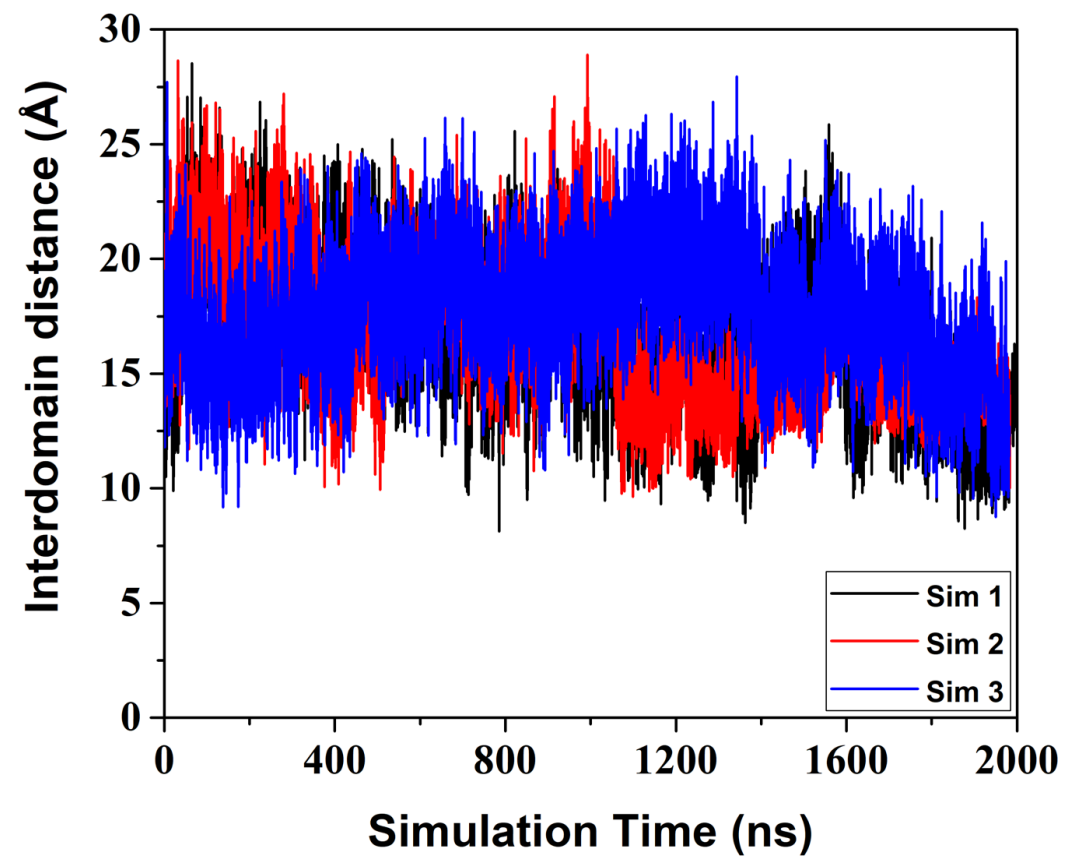

Figure S4: Time course of the interdomain distance (Glu56:CA - Ser128:CA) calculated from three independent LiGaMD trajectories including "Sim 1" (black), "Sim 2" (red) and "Sim 3" (blue). 

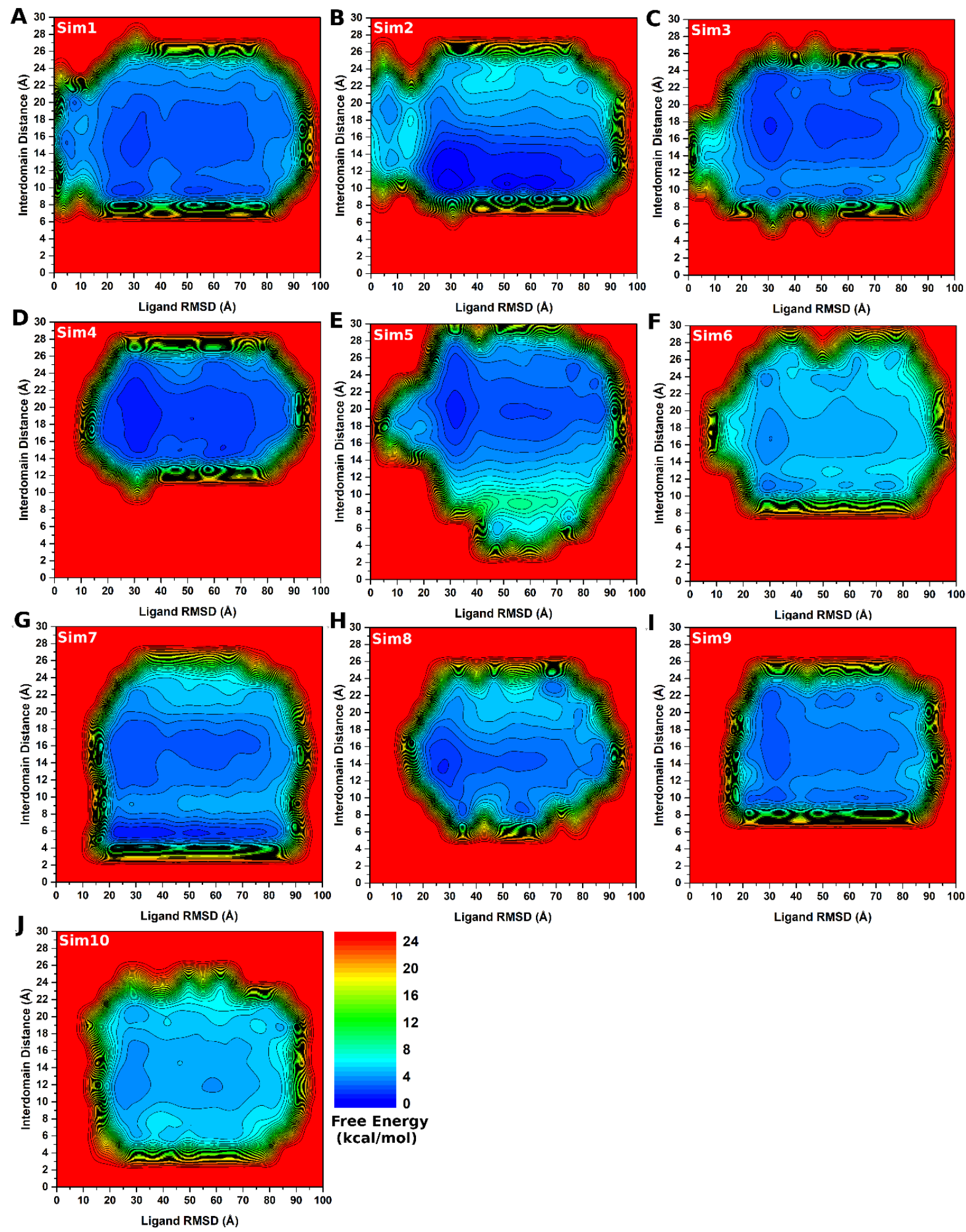

Free Energy

(kcal/mol)

Figure S5: The 2D potential of mean force (PMF) free energy profile of the ligand RMSD and interdomain distance calculated for individual (A) "Sim1", (B) "Sim2", (C) "Sim3" (D) "Sim4", (E) “Sim5”, (F) “Sim6", (G) “Sim7”, (H) “Sim8”, (I) “Sim9” and (J) "Sim10" LiGaMD productio simulations of human ACE2 receptor. 

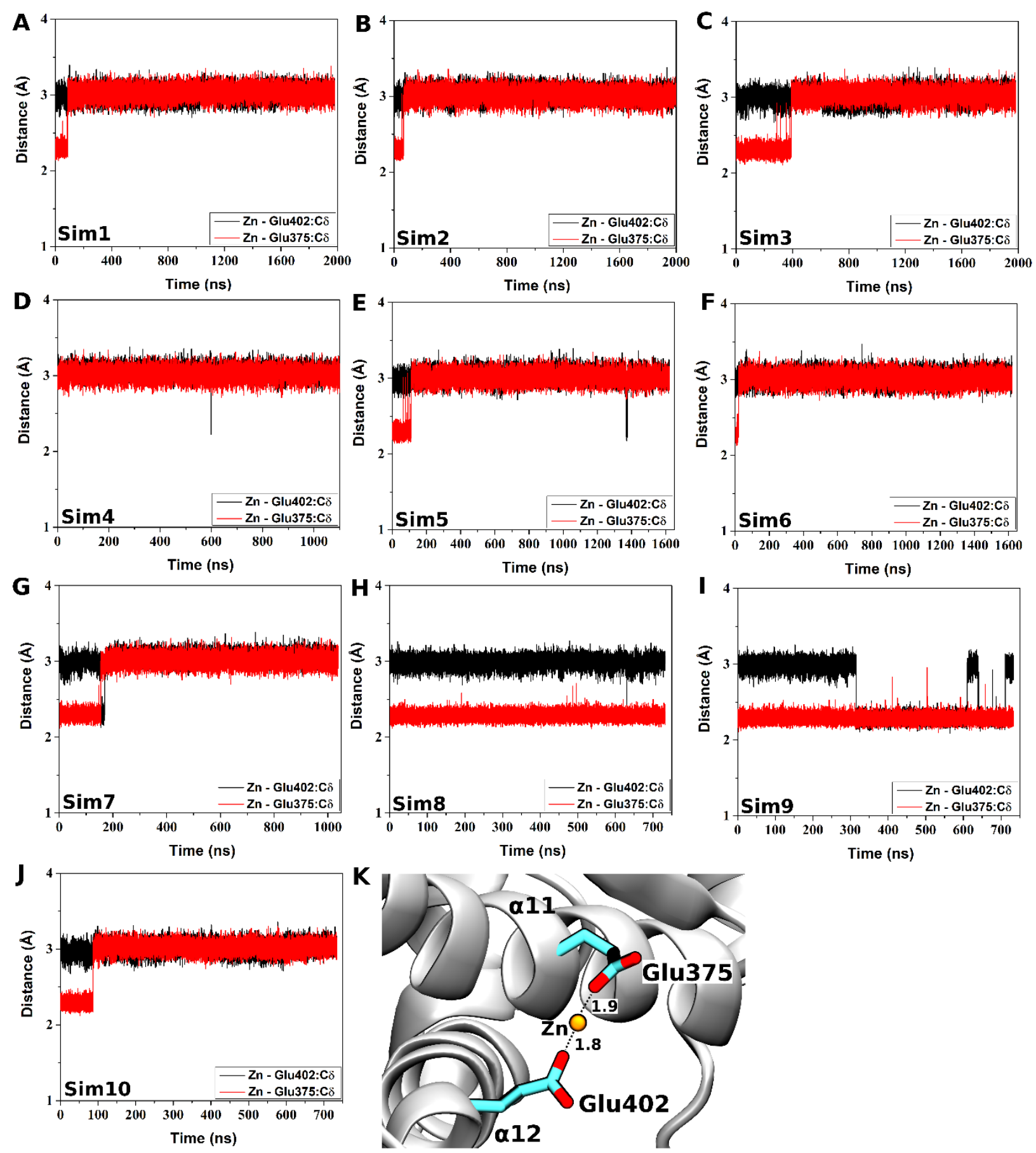

Figure S6: Time course of the distance between $\mathrm{Zn}^{2+}$ and Glu402: $\mathrm{C} \delta$ (black) and Glu375:C $\delta$ (red) calculated from (A) "Sim1", (B) "Sim2", (C) "Sim3", (D) "Sim4", (E) "Sim5”, (F) "Sim6", (G) "Sim7", (H) "Sim8", (I) "Sim9", (J) "Sim10" LiGaMD trajectories of varying lengths ranging $\sim 700-2000$ ns. (K) Zinc (orange ball) near the active site of the ACE2 receptor stabilized by ionic interactions with the Glu375 and Glu402 residues (cyan sticks). The receptor is shown in white ribbons. 


\section{References}

1. Miao, Y.; Feher, V. A.; McCammon, J. A. Gaussian Accelerated Molecular Dynamics: Unconstrained Enhanced Sampling and Free Energy Calculation. J Chem Theory Comput 2015, $11(8), 3584-3595$.

2. Miao, Y.; Bhattarai, A.; Wang, J. Ligand Gaussian accelerated molecular dynamics (LiGaMD): Characterization of ligand binding thermodynamics and kinetics. J Chem Theory Comput 2020, 16 (9), 5526-5547.

3. Vanommeslaeghe, K.; MacKerell, A. D., Jr. CHARMM additive and polarizable force fields for biophysics and computer-aided drug design. Biochimica et biophysica acta 2014.

4. Vanommeslaeghe, K.; Hatcher, E.; Acharya, C.; Kundu, S.; Zhong, S.; Shim, J.; Darian, E.; Guvench, O.; Lopes, P.; Vorobyov, I.; MacKerell, A. D. CHARMM General Force Field: A Force Field for Drug-Like Molecules Compatible with the CHARMM All-Atom Additive Biological Force Fields. J Comput Chem 2010, 31 (4), 671-690.

5. $\quad$ Duan, Y.; Wu, C.; Chowdhury, S.; Lee, M. C.; Xiong, G. M.; Zhang, W.; Yang, R.; Cieplak, P.; Luo, R.; Lee, T.; Caldwell, J.; Wang, J. M.; Kollman, P. A point-charge force field for molecular mechanics simulations of proteins based on condensed-phase quantum mechanical calculations. J Comput Chem 2003, 24 (16), 1999-2012.

6. $\quad$ Cornell, W. D.; Cieplak, P.; Bayly, C. I.; Gould, I. R.; Merz, K. M.; Ferguson, D. M.; Spellmeyer, D. C.; Fox, T.; Caldwell, J. W.; Kollman, P. A. A second generation force field for the simulation of proteins, nucleic acids, and organic molecules (vol 117, pg 5179, 1995). J Am Chem Soc 1996, 118 (9), 2309-2309.

7. $\quad$ Dror, R. O.; Pan, A. C.; Arlow, D. H.; Borhani, D. W.; Maragakis, P.; Shan, Y.; Xu, H.; Shaw, D. E. Pathway and mechanism of drug binding to G-protein-coupled receptors. Proc Natl Acad Sci 2011, 108 (32), 13118-23.

8. Miao, Y.; Bhattarai, A.; Nguyen, A. T. N.; Christopoulos, A.; May, L. T. Structural Basis for Binding of Allosteric Drug Leads in the Adenosine A1 Receptor. Sci. Rep. 2018, 8 (1), 16836.

9. Roux, B. The Calculation of the Potential of Mean Force Using Computer-Simulations. Comput Phys Commun 1995, 91 (1-3), 275-282.

10. Miao, Y.; Sinko, W.; Pierce, L.; Bucher, D.; McCammon, J. A. Improved reweighting of accelerated molecular dynamics simulations for free energy calculation. J Chem Theory Comput 2014, 10 (7), 2677-2689.

11. Towler, P.; Staker, B.; Prasad, S. G.; Menon, S.; Tang, J.; Parsons, T.; Ryan, D.; Fisher, M.; Williams, D.; Dales, N. A. ACE2 X-ray structures reveal a large hinge-bending motion important for inhibitor binding and catalysis. Journal of Biological Chemistry 2004, 279 (17), 17996-18007.

12. Case, D. A.; Cheatham III, T. E.; Darden, T.; Gohlke, H.; Luo, R.; Merz Jr, K. M.; Onufriev, A.; Simmerling, C.; Wang, B.; Woods, R. J. The Amber biomolecular simulation programs. Journal of computational chemistry 2005, 26 (16), 1668-1688.

13. Tian, C.; Kasavajhala, K.; Belfon, K. A.; Raguette, L.; Huang, H.; Migues, A. N.; Bickel, J.; Wang, Y.; Pincay, J.; Wu, Q. ff19SB: Amino-Acid-Specific Protein Backbone Parameters Trained against Quantum Mechanics Energy Surfaces in Solution. Journal of Chemical Theory and Computation 2019, 16 (1), 528-552.

14. Case, D.; Belfon, K.; Ben-Shalom, I.; Brozell, S.; Cerutti, D.; Cheatham, T.; Cruzeiro III, V.; Darden, T.; Duke, R.; Giambasi, G. AMBER 2020. University of California, San Francisco 2020. 
15. Case, D. A.; Cheatham, T. E.; Darden, T.; Gohlke, H.; Luo, R.; Merz, K. M.; Onufriev, A.; Simmerling, C.; Wang, B.; Woods, R. J. The Amber biomolecular simulation programs. $J$ Comput Chem 2005, 26 (16), 1668-1688.

16. Jorgensen, W. L.; Chandrasekhar, J.; Madura, J. D.; Impey, R. W.; Klein, M. L. Comparison of simple potential functions for simulating liquid water. The Journal of chemical physics 1983, 79 (2), 926-935.

17. Humphrey, W.; Dalke, A.; Schulten, K. VMD: Visual molecular dynamics. Journal of Molecular Graphics \& Modelling 1996, 14 (1), 33-38.

18. Roe, D. R.; Cheatham, T. E. PTRAJ and CPPTRAJ: Software for Processing and Analysis of Molecular Dynamics Trajectory Data. J Chem Theory Comput 2013, 9 (7), 30843095. 